\title{
Numerical Existence Proof of Five Solutions for Certain Two-Transistor Circuit Equations
}

\author{
Yusuke NAKAYA ${ }^{\dagger, *}$, Tetsuo Nishi ${ }^{\dagger}$, Shin'ichi OISHI ${ }^{\dagger}$ and Martin Claus ${ }^{\ddagger}$ \\ ${ }^{\dagger}$ Faculty of Science and Engineering, Waseda University \\ 3-4-1 Okubo, Shinjuku-ku, Tokyo 169-8555, Japan \\ *E-mail: nakaya@waseda.jp \\ $\ddagger$ Department of Electrical Engineering and Information Technology \\ Dresden University of Technology, D-01062 Dresden, Germany
}

Received March 31, 2008

Revised December 8, 2008

\begin{abstract}
In this paper, we are concerned with the analysis of two-transistor circuits. Applying technique for the numerical verification, we prove rigorously the existence of five solutions in a two-transistor circuit. The system of equations for a transistor circuit is obtained as nonlinear equations, therefore Krawczyk's method is applied for proving the existence of a solution.
\end{abstract}

Key words: nonlinear equations, existence of solution, Krawczyk's method, transistor circuit

\section{Introduction}

One of the most fundamental problems on the analysis of a transistor circuit (shown in Fig. 1 and hereafter referred to an $m$-transistor circuit) composed of $m$ transistors, linear passive resistors and dc sources is to find the maximum number of solutions of the circuit equation. It was once proposed by the Technical Committee on Nonlinear Circuits and Systems of the IEEE Circuits and Systems Society as a challenging problem. Since nonlinear elements are only transistors, the maximum number of solutions depends on the value of $m$. It is well known that the solution is unique if $m=0$ or $m=1$. However, in spite of long-term efforts we still have no definite answer even for a simple case of $m=2$. The difficulties of the problem can be seen from [1]-[3].

Concerning a two-transistor circuit, by the well-known theorem [4], [5], it has been believed for a long time that the maximum number of solutions is three. The proof [4], [5] given by Lee was too complicated to fully understand. Afterwards it was numerically demonstrated [6]-[8] that several two-transistor circuits have five solutions. Though the possibility of five solutions was numerically predicted [6]-[8], i.e., the existence of solutions was actually shown only by using a circuit simulator, it is not a mathematically guaranteed counter example. The purpose of this paper is to show the existence of five solutions rigorously by using numerical computations with guaranteed accuracy. This implies that Lee's theorem is inaccurate.

For reference we will briefly describe the background of the problem. Since Nielsen and Willson gave a striking theorem [9] on the uniqueness of a solution of 


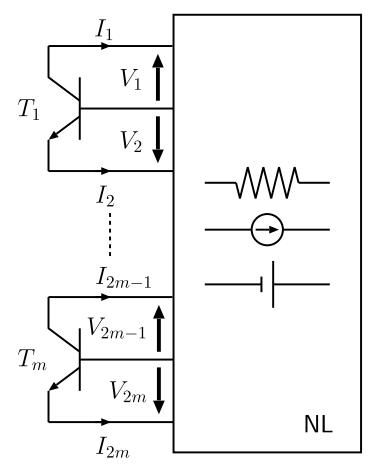

Fig. 1. m-transistor circuit

$m$-transistor circuits, many papers were published on the existence of a solution, the uniqueness of a solution [9]-[11], and the number of solutions [12]-[15]. The circuit equation for an $m$-transistor circuit can be written in general as $F(x)+A x=b$, where $x=\left[x_{1}, x_{2}, \ldots, x_{2 m}\right]^{\mathrm{T}}$ is a variable vector, $F(x)=\left[f_{1}\left(x_{1}\right), f_{2}\left(x_{2}\right), \ldots, f_{2 m}\left(x_{2 m}\right)\right]^{\mathrm{T}}$ is a nonlinear function vector, and $A=\left[a_{i j}\right]$ and $b=\left[b_{i}\right]$ are a constant matrix and a column vector, respectively. Note that $f_{i}\left(x_{i}\right)(i=1,2, \ldots, 2 m)$ is a nonlinear function of the variable $x_{i}$ only. The functions $f_{i}$ and parameters $a_{i j}$ and $b_{i}$ depends on the modelling of a transistor. The typical model of a transistor is the Ebers-Moll model where $f_{i}\left(x_{i}\right)=K\left(e^{\beta_{i} x_{i}}-1\right)\left(K, \beta_{i}>0\right)$ and therefore $f_{i}\left(x_{i}\right)$ is a monotoneincreasing function and $a_{i j}$ and $b_{i}$ are subject to some circuit theoretic restrictions (see Section 3).

The number of solutions depends on various factors such as types of transistors (bipolar junction transistor (BJT) or MOS transistor (MOST)), modelling of a transistor (Ebers-Moll or Gummel-Poon), and the charactersitics of a nonlinear function $f_{i}$ (exponential function, piecewise-linear function, etc.) in transistor models. After the incorrect Lee's theorem, Goldgeisser et al. [6] and Shou et al. [7] showed several circuits possessing five solutions for a MOST circuit and a BTJ circuit respectively by using circuit simulator model. Recently Claus proposed a new analysis method [8] for a two-transistor circuit. Based on this method, Claus showed another MOST circuit and an Ebers-Moll BJT circuit possessing five solutions.

Jitsumatsu and Nishi showed [15] that two-transistor circuits where a transistor is represented by the Ebers-Moll model have at most five solutions if $f_{i}$ are assumed to have ideal diode charactersitics. Jitsumatsu and Nishi also showed [14] that the maximum number of solutions of the two-dimensional nonlinear equation $e^{x_{i}}+a_{i 1} x_{1}+a_{i 2} x_{2}=b_{i}(i=1,2)$ is exactly five if there is no restriction on the real coefficients $a_{i j}$ and $b_{i}$. Therefore, the value "five" may incidentally be a plausable value as the maximum number of solutions for two-transistor circuits, though there is no close relation among the above results. Nishi also shows [13] that on the assumption that only the first and the second derivatives, $f_{i}^{\prime}$ and $f_{i}^{\prime \prime}$, 
are positive, then three-transistor circuits can possess infinitely many solutions (cf. the Nielsen-Willson theorem [9] is obtained on the assumption that only the first derivative $f_{i}^{\prime}$ is positive).

Consequently, although there has been much research on this topic, the solution for $m$-transistor circuits is far from complete.

\section{Numerical verification of a solution for a system of nonlinear equa- tions}

Let $\mathbb{R}, \mathbb{F}, \mathbb{I}$ and $\mathbb{I F}$ be, respectively, the set of real numbers, the set of floating point numbers, the set of intervals on real number, i.e., $\mathbb{I} \mathbb{R}=\{[\underline{x}, \bar{x}] \mid \underline{x} \leq \bar{x}$, $\underline{x}, \bar{x} \in \mathbb{R}\}$, and the set of intervals on floating point number, i.e., $\mathbb{I F}=\{[\underline{x}, \bar{x}] \in \mathbb{I} \mathbb{R} \mid$ $\underline{x}, \bar{x} \in \mathbb{F}\}$. In the following we assume that floating point operations on $\mathbb{F}$ fulfill the IEEE 754 standard for binary floating point numbers [16].

In IEEE 754 some kinds of rounding modes are defined including rounding downwards and rounding upwards, and these two rounding modes are important for rigorous numerical verification. In the following, Round_down and Round_up mean changing the rounding mode to rounding downwards and to rounding upwards respectively.

Consider the numerical verification for a solution of a system of nonlinear equations:

$$
f(x)=0,
$$

where $f: \mathbb{R}^{n} \rightarrow \mathbb{R}^{n}$ is continuously differentiable. We can apply Krawczyk's method which is known as one of efficient methods to prove the existence of a solution for a system of nonlinear equations [17]-[19]. First, we will introduce Krawczyk's method.

For an interval $X \in \mathbb{F}^{n}$ of which the center is $c \in \mathbb{F}^{n}$, an interval matrix $M \in$ $\mathbb{I F}^{n \times n}$ and an interval mapping $K \in \mathbb{I F}^{n}$, which is called the Krawczyk operator, are defined as

$$
\begin{aligned}
M & =E-L^{-1} F^{\prime}(X), \\
K(X) & =c-L^{-1} f(c)+M(X-c)
\end{aligned}
$$

respectively, where $E$ is the $n \times n$ unit matrix, $L \in \mathbb{F}^{n \times n}$ is an approximate matrix of $f^{\prime}(c) \in \mathbb{F}^{n \times n}$, and $F^{\prime}(X) \in \mathbb{I F}^{n \times n}$ is an interval inclusion of $f^{\prime}(X)$, i.e., $f^{\prime}(X) \subseteq F^{\prime}(X)$. Then the following is well-known:

LEMMA 1. If the condition

$$
K(X) \subset X
$$

holds, then there exists a unique solution $x^{*} \in \mathbb{R}^{n}$ of the nonlinear equations (1) in the interval $X$. 
Suppose that an approximate solution $\tilde{x} \in \mathbb{F}^{n}$ of (1) is obtained by some appropriate method. To prove numerically that a true solution exists near $\tilde{x}$, we generate an interval $\tilde{X} \in \mathbb{I F}^{n}$ as

$$
\tilde{X}=\tilde{x}+I
$$

where $I \in \mathbb{I F}^{n}$ is defined as

$$
I=\left(\begin{array}{c}
{[-1,1]} \\
{[-1,1]} \\
\vdots \\
{[-1,1]}
\end{array}\right) e
$$

and $e \in \mathbb{F}$ is chosen as

$$
e=2\left\|\tilde{L}^{-1} f(\tilde{x})\right\|_{\infty}
$$

where $\tilde{L}^{-1} \in \mathbb{F}^{n \times n}$ is an approximate inverse matrix of $f^{\prime}(\tilde{x})$ and $\|x\|_{\infty}$ denotes the infinity norm

$$
\|x\|_{\infty}=\max _{1 \leq i \leq n}\left|x_{i}\right| .
$$

Using the Krawczyk operator (3), we define the operator $H \in \mathbb{F}^{n}$ as

$$
\begin{aligned}
H & =K(\tilde{X})-\tilde{x} \\
& =K(\tilde{x}+I)-\tilde{x} \\
& =-\tilde{L}^{-1} f(\tilde{x})+\left(E-\tilde{L}^{-1} F^{\prime}(\tilde{x}+I)\right) I .
\end{aligned}
$$

Then the condition (4) is equivalent to

$$
H \subset I
$$

We therefore see by Lemma 1 that if the condition (10) holds, then the interval $\tilde{X}$ contains a true solution of the nonlinear equation (1). So we will check using numerical computations with guaranteed accuracy whether the condition (10) is satisfied or not.

We now implement Krawczyk's method by interval arithmetic on doubles satisfying IEEE 754 standard. Assume that $f(\tilde{x}) \in \mathbb{I F}^{n}$ and $F^{\prime}(\tilde{x}+I) \in \mathbb{I F}^{n \times n}$ are calculated in advance as intervals with guaranteed accuracy as

$$
f(\tilde{x}) \subseteq\left[f_{c}-f_{r}, f_{c}+f_{r}\right]
$$

and

$$
F^{\prime}(\tilde{x}+I) \subseteq\left[F_{c}^{\prime}-F_{r}^{\prime}, F_{c}^{\prime}+F_{r}^{\prime}\right]
$$


respectively, where $f_{c} \in \mathbb{F}^{n}, F_{c}^{\prime} \in \mathbb{F}^{n \times n}$ are the center of the intervals, and $f_{r} \in \mathbb{F}^{n}$, $F_{r}^{\prime} \in \mathbb{F}^{n \times n}$ are the radius of the intervals. From (9), we need to calculate $-\tilde{L}^{-1} f(\tilde{x})$ and $E+\left(-\tilde{L}^{-1}\right) F^{\prime}(\tilde{x}+I)$ at first. By applying the interval arithmetic these values are calculated as follows respectively:

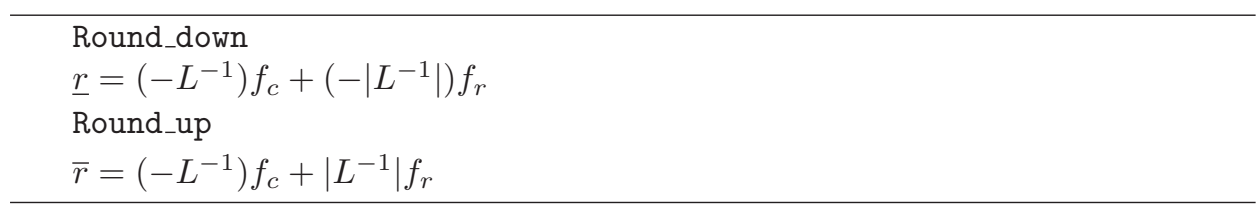

Round_down

$\underline{M}=E+\left(-L^{-1}\right) F_{c}^{\prime}+\left(-\left|L^{-1}\right|\right) F_{r}^{\prime}$

Round_up

$\bar{M}=E+\left(-L^{-1}\right) F_{c}^{\prime}+\left|L^{-1}\right| F_{r}^{\prime}$

then $-\tilde{L}^{-1} f(\tilde{x}) \subseteq[\underline{r}, \bar{r}]$ and $E+\left(-\tilde{L}^{-1}\right) F^{\prime}(\tilde{x}+I) \subseteq[\underline{M}, \bar{M}]$ hold. Next we have to implement the calculation of $\left(E-\tilde{L}^{-1} F^{\prime}(\tilde{x}+I)\right) I$. Since $I$ is given by $(6)$, this part can be realized as

$$
\begin{aligned}
& \text { Round_up } \\
& M_{\max }=\max \{|\underline{M}|,|\bar{M}|\} \\
& q=M_{\max } e
\end{aligned}
$$

and $\left(E-\tilde{L}^{-1} F^{\prime}(\tilde{x}+I)\right) I \subseteq[-q, q]$ holds. Finally we calculate the value of operator $H$ as

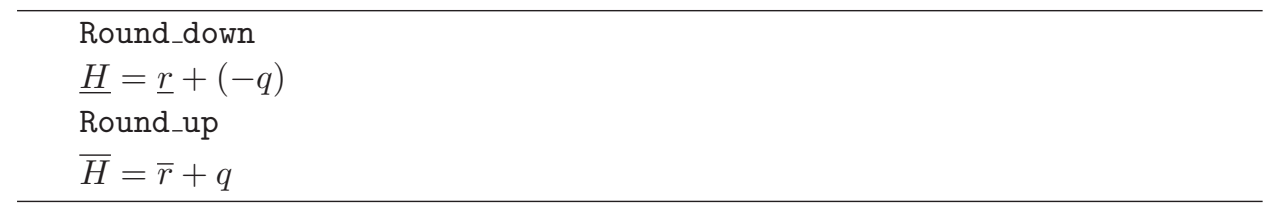

From this algorithm, rigorous range of $H$ can be evaluated, and the following result holds:

$$
H \subset[\underline{H}, \bar{H}] .
$$

Therefore, by collecting the above results, Krawczyk's method is implemented by the following algorithm: 
Algorithm 1.

Round_down

$\underline{r}=\left(-L^{-1}\right) f_{c}+\left(-\left|L^{-1}\right|\right) f_{r}$

$\underline{M}=E+\left(-L^{-1}\right) F_{c}^{\prime}+\left(-\left|L^{-1}\right|\right) F_{r}^{\prime}$

Round_up

$\bar{r}=\left(-L^{-1}\right) f_{c}+\left|L^{-1}\right| f_{r}$

$\bar{M}=E+\left(-L^{-1}\right) F_{c}^{\prime}+\left|L^{-1}\right| F_{r}^{\prime}$

$M_{\max }=\max \{|\underline{M}|,|\bar{M}|\}$

$q=M_{\max } e$

$\bar{H}=\bar{r}+q$

Round_down

$\underline{H}=\underline{r}+(-q)$

3. Application of Krawczyk's method to numerical verification of solutions of two-transistor circuits

We will examine the flip-flop circuit [8] shown in Fig. 2 by using numerical computations with guaranteed accuracy. As a result, we will rigorously prove that the circuit has five solutions with certainty.

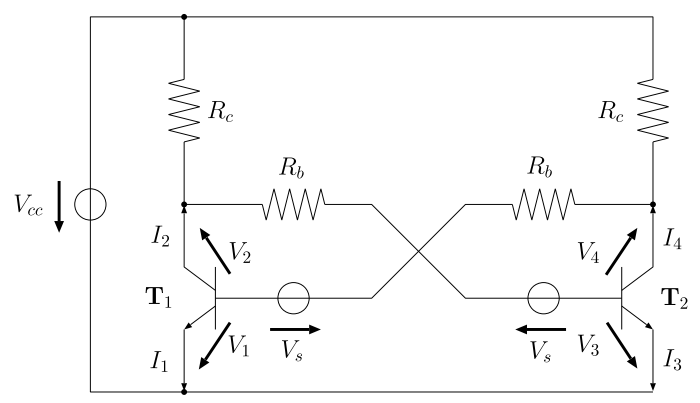

Fig. 2. Flip-flop circuit

The Ebers-Moll model of the transistor in Fig. 3 (a) is represented in Fig. 3 (b). The terminal currents $I_{i}(i=1,2,3,4)$ of transistors are given as

$$
\begin{aligned}
{\left[\begin{array}{l}
-I_{k+1} \\
-I_{k+2}
\end{array}\right] } & =\left[\begin{array}{cc}
1 & -\alpha_{r} \\
-\alpha_{f} & 1
\end{array}\right]\left[\begin{array}{l}
f_{k+1}\left(V_{k+1}\right) \\
f_{k+2}\left(V_{k+2}\right)
\end{array}\right], \\
{\left[\begin{array}{l}
f_{k+1}\left(V_{k+1}\right) \\
f_{k+2}\left(V_{k+2}\right)
\end{array}\right] } & =\left[\begin{array}{c}
\frac{I_{s}}{\alpha_{f}}\left(e^{\frac{V_{k+1}}{V_{T}}}-1\right) \\
\frac{I_{s}}{\alpha_{r}}\left(e^{\frac{V_{k+2}}{V_{T}}}-1\right)
\end{array}\right] \quad(k=0,1) .
\end{aligned}
$$




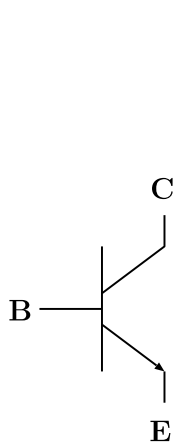

(a) Transistor

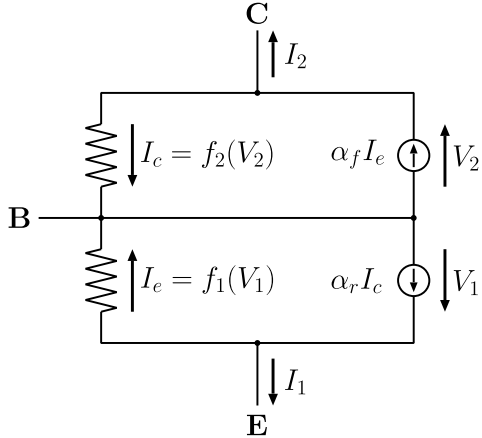

(b) Ebers-Moll model

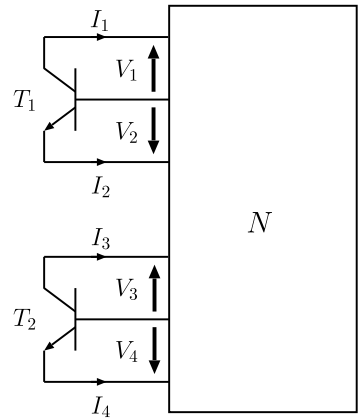

(c) 4-port $N$

Fig. 3. Ebers-Moll model and 4-port $N$

The linear 4-port $N$ obtained from the total circuit by removing two transistors is depicted in Fig. 3 (c). Then the charactersitics of $N$ can be represented by using the conductance matrix $G$ of the 4-port as:

$$
I=G V+J
$$

where $I=\left[I_{1}, I_{2}, I_{3}, I_{4}\right]^{\mathrm{T}}, V=\left[V_{1}, V_{2}, I_{3}, I_{4}\right]^{\mathrm{T}}$,

$$
\begin{aligned}
G & =\left[\begin{array}{cccc}
2 G_{b}+G_{c} & -\left(G_{b}+G_{c}\right) & -2 G_{b} & G_{b} \\
-\left(G_{b}+G_{c}\right) & G_{b}+G_{c} & G_{b} & 0 \\
-2 G_{b} & G_{b} & 2 G_{b}+G_{c} & -\left(G_{b}+G_{c}\right) \\
G_{b} & 0 & -\left(G_{b}+G_{c}\right) & G_{b}+G_{c}
\end{array}\right], \\
J & =\left[\begin{array}{c}
G_{c} V_{c c} \\
G_{b} V_{s}-G_{c} V_{c c} \\
G_{c} V_{c c} \\
G_{b} V_{s}-G_{c} V_{c c}
\end{array}\right],
\end{aligned}
$$

$G_{b}=1 / R_{b}$ and $G_{c}=1 / R_{c}$. Substituting (18) into (19), we have the circuit equation as follows:

$$
T f(V)+G V+J=0
$$

where

$$
T=\left[\begin{array}{cccc}
1 & -\alpha_{r} & 0 & 0 \\
-\alpha_{f} & 1 & 0 & 0 \\
0 & 0 & 1 & -\alpha_{r} \\
0 & 0 & -\alpha_{f} & 1
\end{array}\right], \quad f(V)=\left[\begin{array}{c}
\frac{I_{s}}{\alpha_{f}}\left(e^{\frac{V_{1}}{V_{T}}}-1\right) \\
\frac{I_{s}}{\alpha_{r}}\left(e^{\frac{V_{2}}{V_{T}}}-1\right) \\
\frac{I_{s}}{\alpha_{f}}\left(e^{\frac{V_{3}}{V_{T}}}-1\right) \\
\frac{I_{s}}{\alpha_{r}}\left(e^{\frac{V_{4}}{V_{T}}}-1\right)
\end{array}\right]
$$


Let the values of $R_{b}, R_{c}$ and $V_{c c}$ be $10 \mathrm{k} \Omega, 5 \mathrm{k} \Omega$ and $-5 \mathrm{~V}$ respectively and let the parameters of transistors be chosen as $\alpha_{f}=0.99, \alpha_{r}=0.5$. The dc source value $V_{s}$ is chosen as a parameter. We consider the following two cases: (a) $I_{s}=10^{-9}(\mathrm{~A})$, $V_{T}=0.053(\mathrm{~V}),(\mathrm{b}) I_{s}=10^{-6}(\mathrm{~A}), V_{T}=0.102(\mathrm{~V})$.

On the first step, we calculate approximate solutions of the equation (21) for various values of $V_{s}$ by usual numerical calculation. We then obtain the bifurcation diagrams in Figs. 4 (for cases (a)) and 5 (for cases (b)), respectively. The approximate solutions of $V_{1}$ for $V_{s}=V_{s 0}$, for example, can be found as the intersection of the curves of $V_{1}$ and the vertical line $V_{s}=V_{s 0}$. Seeing these bifurcation diagrams, in cases (b), we may surmise that for values in the interval $[-0.46,-0.39]$ of $V_{s}$, there exist five solutions of the equation (21). However, this is not logically correct in most cases. We will proceed to prove the existence of solutions in the 4-dimensional space.
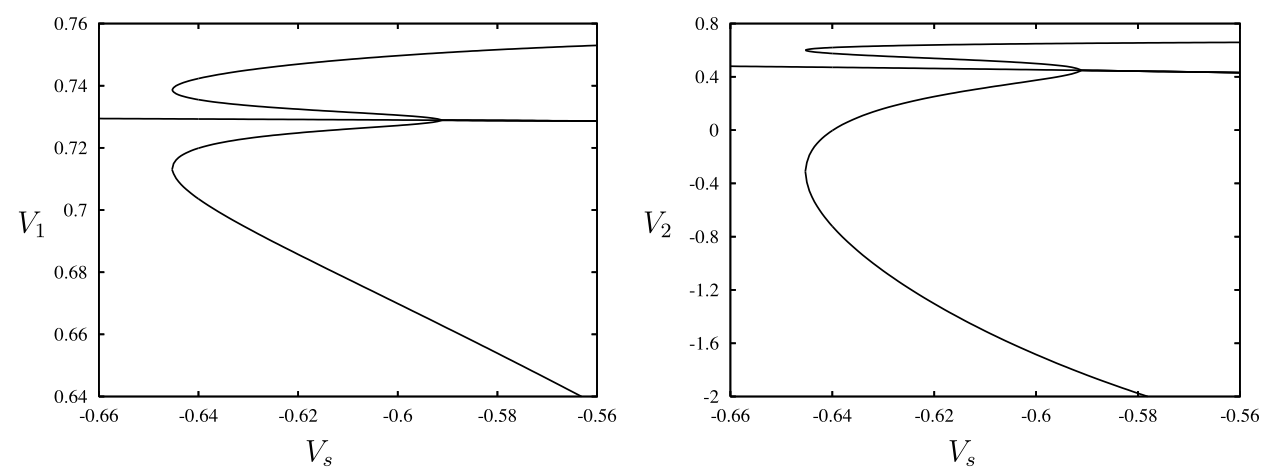

Fig. 4. Bifurcation diagrams for the case (a)
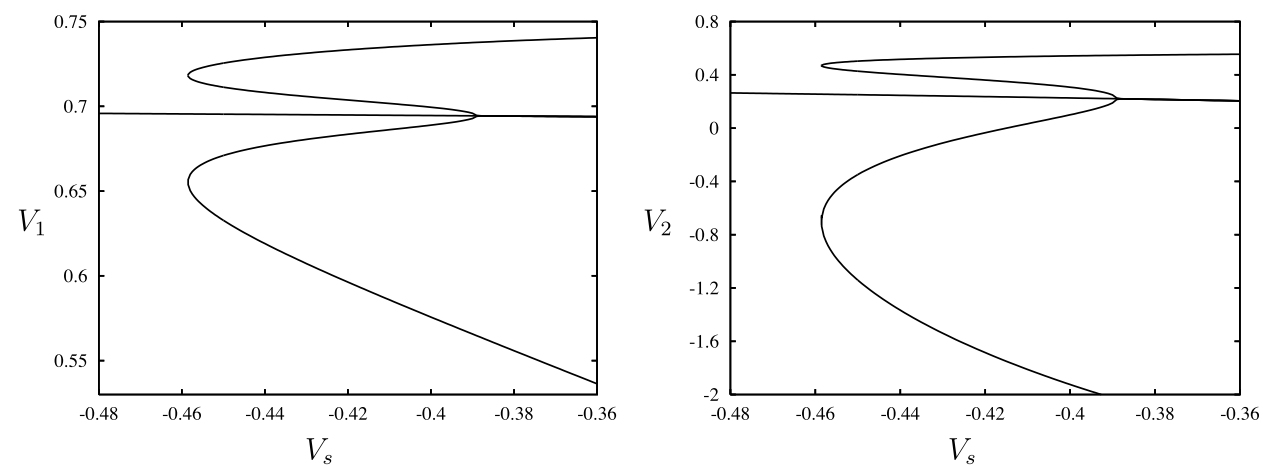

Fig. 5. Bifurcation diagrams for the case (b)

On the second step, we will examine whether five solution surely exist by applying Krawczyk's method to the nonlinear equation (21). We choose the parameter $V_{s}$ as $V_{s}=-0.64(\mathrm{~V})$ for case (a) and $V_{s}=-0.44(\mathrm{~V})$ for case (b) for which five solutions are expected from Figs. 4 and 5. For each approximate solution obtained 
previously, we generate the interval by (5) and attempt a numerical verification of the solution by applying the operator $H$ and verifying that the condition (10) is satisfied.

Tables 1 and 2 show the final results. These tables show that five verified solutions are obtained for each cases of (a) and (b). Thus we rigorously proved that the circuit shown in Fig. 2 have at least five operating points.

Table 1. Verified solutions of case (a) on $V_{s}=-0.64(\mathrm{~V})$

\begin{tabular}{|c||c|c|c|c|}
\hline No. & $V_{1}$ & $V_{2}$ & $V_{3}$ & $V_{4}$ \\
\hline \hline$\# 1$ & {$[0.7423164,0.7423165]$} & {$[0.6198872,0.6198873]$} & {$[0.7035896,0.7035897]$} & {$[-0.7218072,-0.7218071]$} \\
$\# 2$ & {$[0.7355125,0.7355126]$} & {$[0.5755529,0.5755530]$} & {$[0.7199016,0.7199017]$} & {$[-0.0033359,-0.0033358]$} \\
$\# 3$ & {$[0.7292896,0.7292897]$} & {$[0.4714551,0.4714552]$} & {$[0.7292896,0.7292897]$} & {$[0.4714551,0.4714552]$} \\
$\# 4$ & {$[0.7199016,0.7199017]$} & {$[-0.0033359,-0.0033358]$} & {$[0.7355125,0.7355126]$} & {$[0.5755529,0.5755530]$} \\
$\# 5$ & {$[0.7035896,0.7035897]$} & {$[-0.7218072,-0.7218071]$} & {$[0.7423164,0.7423165]$} & {$[0.6198872,0.6198873]$} \\
\hline
\end{tabular}

Table 2. Verified solutions of case (b) on $V_{s}=-0.44(\mathrm{~V})$

\begin{tabular}{|c||c|c|c|c|}
\hline No. & $V_{1}$ & $V_{2}$ & $V_{3}$ & $V_{4}$ \\
\hline \hline$\# 1$ & {$[0.7288801,0.7288802]$} & {$[0.5163018,0.5163019]$} & {$[0.6190239,0.6190240]$} & {$[-1.3666412,-1.3666411]$} \\
$\# 2$ & {$[0.7081693,0.7081694]$} & {$[0.4034650,0.4034651]$} & {$[0.676678,0.676679]$} & {$[-0.2082450,-0.2082449]$} \\
$\# 3$ & {$[0.6952142,0.6952143]$} & {$[0.2462035,0.2462036]$} & {$[0.6952142,0.6952143]$} & {$[0.2462035,0.2462036]$} \\
$\# 4$ & {$[0.676678,0.676679]$} & {$[-0.2082450,-0.2082449]$} & {$[0.7081693,0.7081694]$} & {$[0.4034650,0.4034651]$} \\
$\# 5$ & {$[0.6190239,0.6190240]$} & {$[-1.3666412,-1.3666411]$} & {$[0.7288801,0.7288802]$} & {$[0.5163018,0.5163019]$} \\
\hline
\end{tabular}

\section{Conclusion}

We have shown rigorously the existence of five solutions in a two-transistor circuit by applying numerical computations with guaranteed accuracy. From this result, we proved surely that the maximum number of solutions for two-transistor circuits is at least five.

\section{References}

[1] L. Kronenberg, L. Trajkovic and W. Mathis, Finding dc operating points: Limitations of topological and determinant criteria. Proc. of International Symposium on Nonlinear Theory and Its Applications (NOLTA 2000), Dresden, Germany, 2000, 209-212.

[ 2 ] L. Kronenberg, L. Trajkovic and W. Mathis, Analysis of feedback structures and effect on multiple dc operating points. European Circuit Theory and Design Conference, Stresa, Italy, 1999, 683-686.

[ 3 ] L. Kronenberg, W. Mathis and L. Trajkovic, Limitations of criteria for testing transistor circuits for multiple dc operating points. Proc. 43rd Midwest Symposium on Circuits and Systems (MWSCAS 2000), Lansing, MI, 2000, 1156-1159.

[ 4 ] B.G. Lee and A.N. Willson, Number of dc solutions of two-transistor circuits containing feedback structures. Dissertation, Los Angeles, CA: Univ. of Calif., Los Angeles, El. Eng. Dept., 1982.

[ 5 ] B.G. Lee and A.N. Willson, All two-transistor circuits possess at most three dc operating equilibrium points. Proc. 26th Midwest Symp. Circuits and Systems, Puebla, Mexico, 1983, 504-507.

[6] L.B. Goldgeisser and M.M. Green, Some two-transistor circuits possess more than three operation points. Proc. IEEE International Symposium on Circuits and Systems, 1999, 1002-1006. 
[ 7 ] X. Shou, L.B. Goldgeisser and M.M. Green, A methodology for constructing two-transistor multistable circuits. Proc. IEEE International Symposium on Circuits and Systems, 2001, $377-380$

[ 8 ] M. Claus, Geometrical analysis of two-transistor circuits with more than three operating points. Proc. of European Conference on Circuit Theory and Design (ECCTD 2005), 2005.

[9] R. Nielsen and A.N. Willson, A fundamental result concerning the topology of transistor circuits with multiple equilibria. Proc. IEEE, 68 (1980), 196-208.

[10] T. Nishi and L.O. Chua, Topological criteria for nonlinear resistive circuits containing controlled sources to have a unique solution. IEEE Trans. on Circuits and Systems, CAS-31 (1984), 722-741.

[11] M. Fosseprez, M. Hasler and C. Schnetzer, On the number of solutions of piecewise linear resistive circuits. IEEE Trans. Circuits and Systems, 36 (1989), 393-402.

[12] T. Nishi and Y. Kawane, On the number of solutions of nonlinear resistove circuits. IEICE Trans. on Fundamentals, E74 (1991).

[13] T, Nishi, A transistor circuit can possess infinitely many solutions under the assumption that the first and the second derivatives of the $v-i$ curves of nonlinear resistors are positive. Proc. ISCAS '96, Atlanta, 1996.

[14] Y. Jitsumatsu and T. Nishi, Maximum number of solutions for a kind of nonlinear simultaneous equations with two variables. IEICE Transactions on Fundamentals, 82-A (1999), 1175-1176 (in Japanese).

[15] Y. Jitsumatsu and T. Nishi, On the number of solutions for a class of piecewise-linear equations related to transistor circuits. IEICE Trans. on Fundamentals, E84-A (2001), $2221-2229$.

[16] ANSI/IEEE 754-1985, Standard for Binary Floating-Point Arighmetic. 1985.

[17] R. Krawczyk, Newton-Algorithm zur Bestimmung von Nullstellen mit Fehleshranken. Computing, 4 (1969), 187-201.

[18] R.E. Moore, A test for existence of solutions for non-linear systems. SIAM J. Numerical Analysis, 14 (1977), 611-615.

[19] A. Neumaier, Interval methods for systems of equations. Encyclopedia of Mathematics and Its Applications, Cambridge University Press, 1990. 